\title{
Atomically Precise Copper Cluster with Intensely Near-Infrared Luminescence and Its Mechanism
}

\author{
Hao Li,${ }^{\dagger}$ Hongsheng Zhai, ${ }^{\S}$ Chuanjun Zhou, ${ }^{\dagger}$ Yongbo Song $,{ }^{*}, \dagger$ Feng Ke,${ }^{\dagger}$ Wen Wu Xu${ }^{*, \#}$ and \\ Manzhou Zhu*,†
}

Department of Chemistry and Centre for Atomic Engineering of Advanced Materials, Anhui Province Key Laboratory of Chemistry for Inorganic/Organic Hybrid Functionalized Materials, Key Laboratory of Structure and Functional Regulation of Hybrid Materials of Ministry of Education, Anhui University, Hefei, 230601, People's Republic of China.

\$Spectral Measurement and Application of Infrared Material Key Laboratory of Henan Province, School of Physics, Henan Normal University, Xinxiang 453007, People’s Republic of China.

\#Department of Physics, School of Physical Science and Technology, Ningbo University, Ningbo 315211, People's Republic of China.

\section{Materials}

All reagents are commercially available and used directly without further purification. $\mathrm{CuCl}$ (99.8\%), 4-tert-butylthiophenol (98\%), tetraoctylammonium bromide (TOAB, 98\%), triphenylphosphine $\left(\mathrm{PPh}_{3}, 99 \%\right)$, sodium borohydride $(99.99 \%)$, chloroform (HPLC grade, $\geq 99.9 \%$ ), methanol (HPLC grade, $\geq 99.9 \%$ ), n-hexane (HPLC grade, $\geq$ 99.9\%). All glassware was cleaned with aqua regia $\left(\mathrm{HCl}: \mathrm{HNO}_{3}=3: 1, v: v\right)$ and washed thoroughly with pure water before used.

\section{Synthesis of the $\left[\mathrm{Cu} u_{11}\left(\mathrm{TBBT}_{9}\right)_{9}\left(\mathrm{PPh}_{3}\right)_{6}\right]\left(\mathrm{SbF}_{6}\right)_{2}$ cluster.}

The whole reaction process is carried out at room temperature.100 $\mathrm{mg}$ tetraoctylammonium bromide and $40 \mathrm{mg} \mathrm{CuCl}$ were dissolved in a mixed solvent of chloroform $(15 \mathrm{~mL})$ and methanol $(10 \mathrm{~mL})$ in a $100 \mathrm{~mL}$ round bottom flask at vigorously stirred $(\sim 1200 \mathrm{rpm})$. After that, $100 \mathrm{mg}$ triphenylphosphine was added into above solution. After $30 \mathrm{mins}, 70 \mathrm{uL}$ 4-tert-butylbenzenethiol was directly added to the above solution. 30 mins later, $5 \mathrm{~mL}$ aqueous solution of $50 \mathrm{mg} \mathrm{NaBH} 4$ was rapidly added. 24 hours later, the aqueous solution was removed, and $100 \mathrm{mg} \mathrm{NaSbF}_{6}$ dissolved in $5 \mathrm{~mL}$ methanol was added into the organic solution. The organic solution was dried by rotary evaporation and washed several times with methanol to remove the residual ligands and by-products. Finally, the yellow crystal was obtained by n-hexane diffusion into the chloroform solution for one week. 


\section{Synthesis of the $\mathrm{Cu}(\mathrm{I})-\mathrm{SR}$ complex.}

$10 \mathrm{mg} \mathrm{CuCl}_{2}$ dissolved in $10 \mathrm{~mL}$ methanol and $100 \mu \mathrm{L}$ 4-tert-butylthiophenol were mixed in $25 \mathrm{~mL}$ round bottom flask under vigorously stirring. After 10 mins, the organic solution was removed by centrifugation. And then, the product was washed several times with methanol to remove the residual ligands. Finally, the $\mathrm{Cu}(\mathrm{I})$-SR complex was collected by centrifugation

\section{Characterization}

Single crystal $X$-ray diffractometer. Single crystal X-ray diffraction data was collected on a Bruker D8 Venture diffractometer with the Ga Kl $\alpha$ X-ray source $(\lambda=1.34139 \AA)$. A yellowish needle-like crystal with dimensions $0.1 \times 0.03 \times 0.02 \mathrm{~mm}$ was mounted. Data collection was performed at $170 \mathrm{~K}$. Using Olex $2,{ }^{1}$ the structure was solved with the ShelXT ${ }^{2}$ structure solution program using Intrinsic Phasing and refined with the ShelXL ${ }^{3}$ refinement package using Least Squares minimisation. All the $\mathrm{Cu}$ and $\mathrm{S}$ atoms were found directly. Remaining non-hydrogen atoms were generated via subsequent difference Fourier syntheses. All the non-hydrogen atoms were refined anisotropically. All the hydrogen atoms were set in geometrically calculated positions and refined isotropically using a riding model. The diffuse electron densities resulting from the residual solvent molecules were removed from the data set using the SQUEEZE routine of PLATON and refined further using the data generated.

Electrospray ionization (ESI) mass spectrum was performed on Waters XEVO G2XS QTof mass spectrometer. The samples are dissolved in a mixture solution of $\mathrm{CH}_{2} \mathrm{Cl}_{2} / \mathrm{CH}_{3} \mathrm{OH}(\mathrm{v}: \mathrm{v}=1: 1)$, which is directly infused into the chamber at $10 \mu \mathrm{L} / \mathrm{min}$ with positive mode.

Thermogravimetric analysis (TGA) was carried out on a thermogravimetric analyzer (DTG-60H, Shimadzu Instruments Inc., Japan) at a heating rate of $10 \mathrm{~K} \cdot \mathrm{min}^{-1}$ in $\mathrm{N}_{2}$ atmosphere.

${ }^{1}$ HNMR were performed on a Bruker Avance ${ }^{\mathrm{TM}}$ II 400 NMR instrument. The data was collected with $\sim 5 \mathrm{mg}$ clusters dissolved in $0.6 \mathrm{~mL} \mathrm{CD}_{2} \mathrm{Cl}_{2}$.

Inductively coupled plasma mass spectrometry (ICP-MS) was performed by Thermo iCAP QICP-MS.

Powder X-ray diffraction (XRD) pattern was obtained on SmartLab 9KW with $\mathrm{Cu} \mathrm{K \alpha}$ radiation.

X-ray photoelectron spectroscopy (XPS) measurements were performed on Thermo ESCALAB 250 equipped with a monochromated Al Ka (1486.8 eV) $150 \mathrm{~W}$ X-ray source, $0.5 \mathrm{~mm}$ circular spot size, a flood gun to counter charging effects, and the 
analysis chamber base pressure lower than $1 \times 10^{-9}$ mbar; data were collected with FAT $=20 \mathrm{eV}$.

UV-vis absorption spectra were recorded on Agilent 8453 spectrophotometer. Crystals are dissolved in solution.

Photoluminescence spectra were measured on a HORIBA JY Fluorolog 3-tau.

Absolute quantum yields (QYs) and emission lifetime were measured on a HORIBA FluoroMax-4P.

Calculation. All geometry optimizations were performed using density functional theory (DFT) as implemented in the Amsterdam Density Functional (ADF) 2017 package. ${ }^{4,5}$ The BP86 exchange-correlation functional was used for these calculations. ${ }^{6,7}$ A TZP basis set and a large frozen electron core were used to ascertain completeness. Generalized gradient approximation (GGA) functionals have been employed successfully in calculating geometric and electronic structures. All structures are optimized in the gas phase.

\section{Reference:}

(1) Dolomanov, O.V., Bourhis, L.J., Gildea, R.J, Howard, J.A.K. \& Puschmann, H. (2009), J. Appl. Cryst. 42, 339-341.

(2) Sheldrick, G.M. (2015). Acta Cryst. A71, 3-8.

(3) Sheldrick, G.M. (2015). Acta Cryst. C71, 3-8.

(4) te Velde, G.; Bickelhaupt, F. M.; Baerends, E. J.; Fonseca Guerra, C.; van Gisbergen, S. J. A.; Snijders, J. G.; Ziegler, T. Chemistry with ADF. J. Comput. Chem. 2001, 22, 931-967.

(5) Aerends, E. J.; et al. ADF2017, Scm, Theoretical Chemistry;Vrije Universiteit: Amsterdam (https://www.scm.com).

(6) Becke, A. D. Density-Functional Exchange-Energy Approximation with Correct Asymptotic Behavior. Phys. Rev. A: At., Mol.,Opt. Phys. 1988, 38, 3098-3100.

(7) Perdew, J. P. Density-Functional Approximation For the Correlation Energy of the Inhomogeneous Electron Gas. Phys. Rev. B: Condens. Matter Mater. Phys. 1986, $33,8822-8824$. 


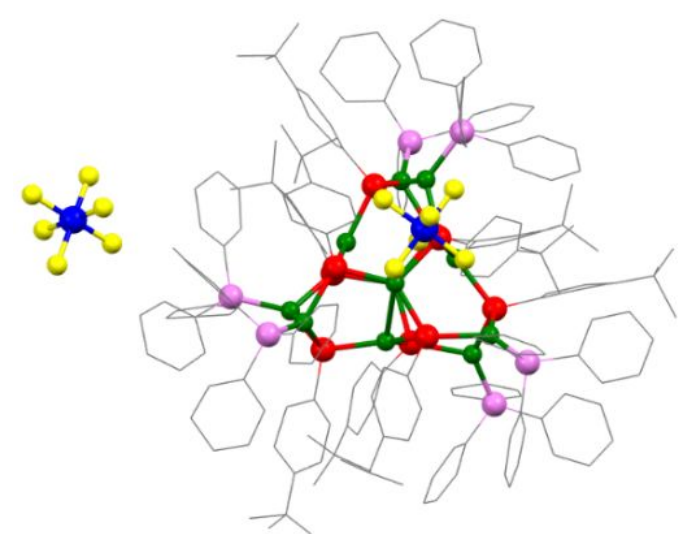

Figure S1. Two SbF ${ }^{6-}$ ions accompanied with $\mathbf{C u}_{11}$ cluster.

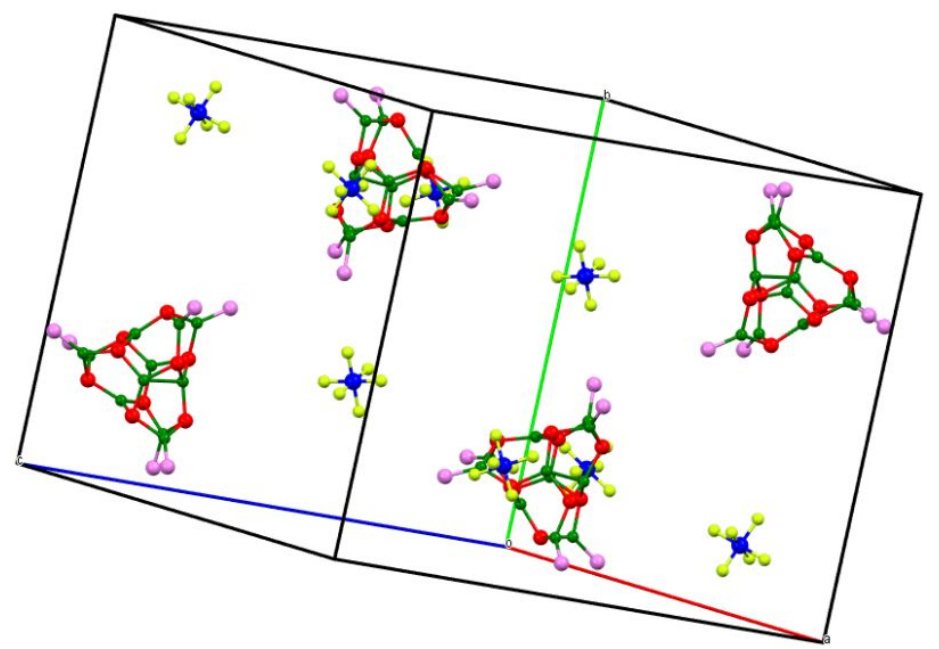

Figure S2. Two pairs of enantiomers in unit cell.
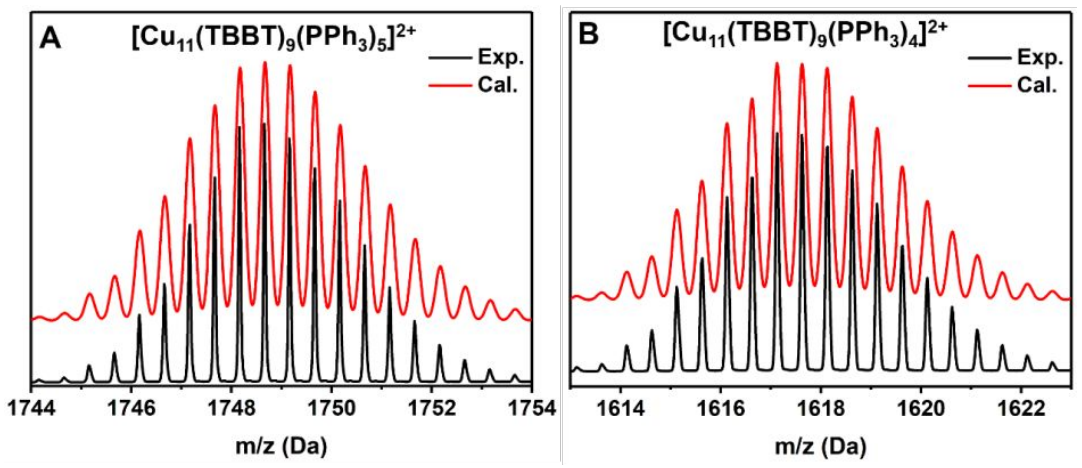

Figure S3. Comparison of the calculated (red) and experimental (black) isotope distribution patterns of (A) $\left[\mathrm{Cu}_{11}(\mathrm{TBBT})_{9}\left(\mathrm{PPh}_{3}\right)_{5}\right]^{2+}$ and (B) $\left[\mathrm{Cu}_{11}(\mathrm{TBBT})_{9}\left(\mathrm{PPh}_{3}\right)_{4}\right]^{2+}$. 


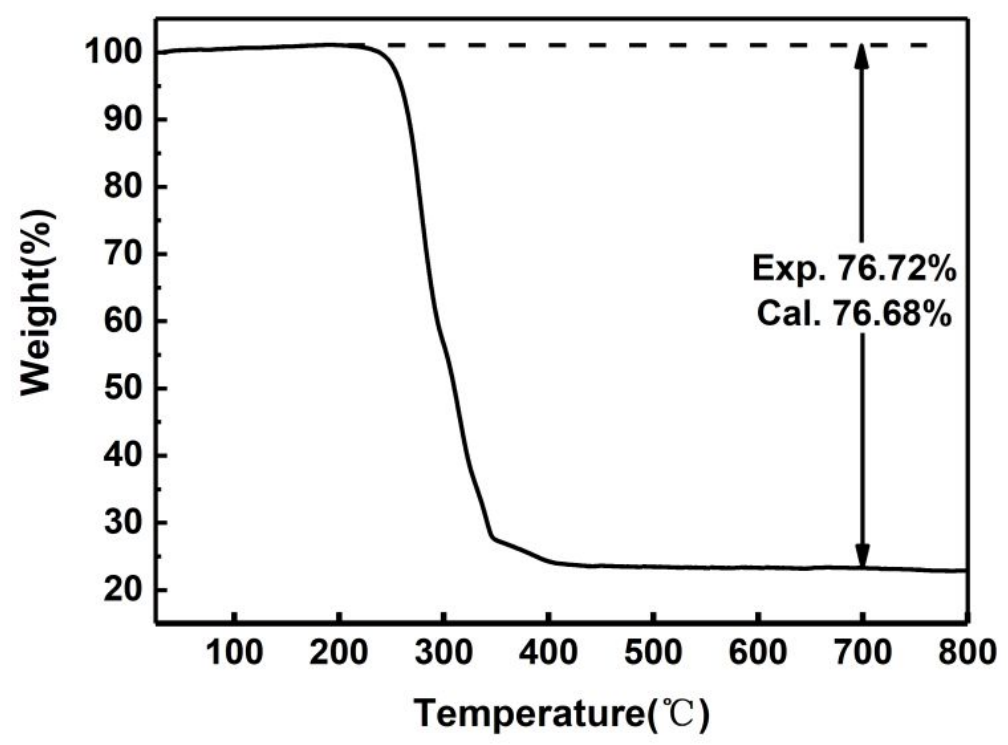

Figure S4. TGA curve of $\mathbf{C} \mathbf{u}_{11}$ cluster.

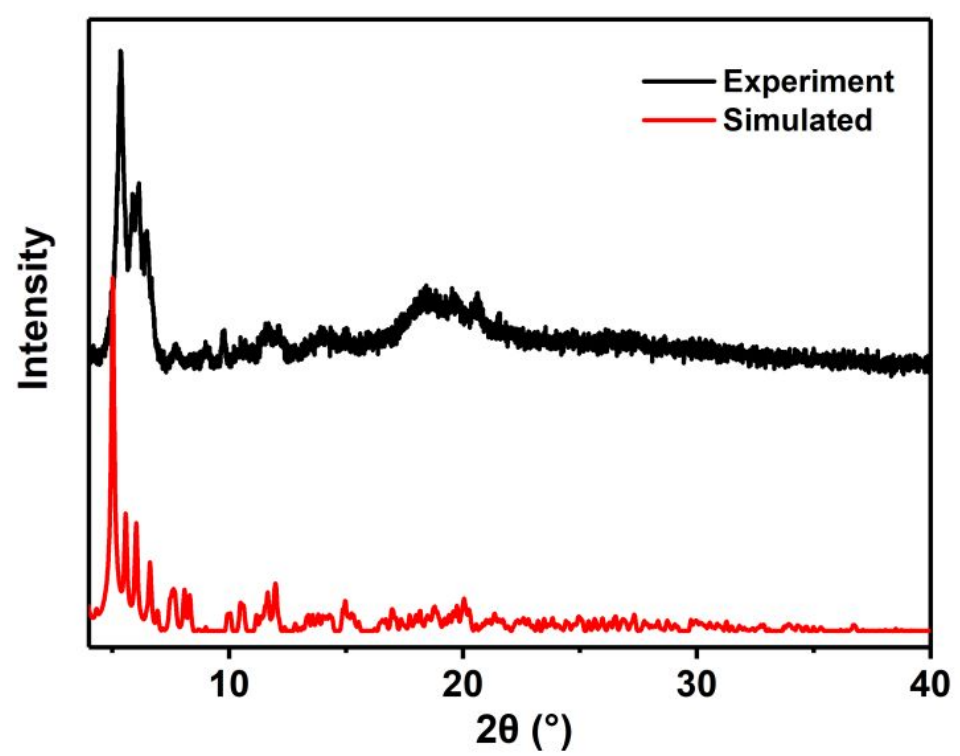

Figure S5. PXRD of $\mathbf{C u}_{11}$ cluster. 


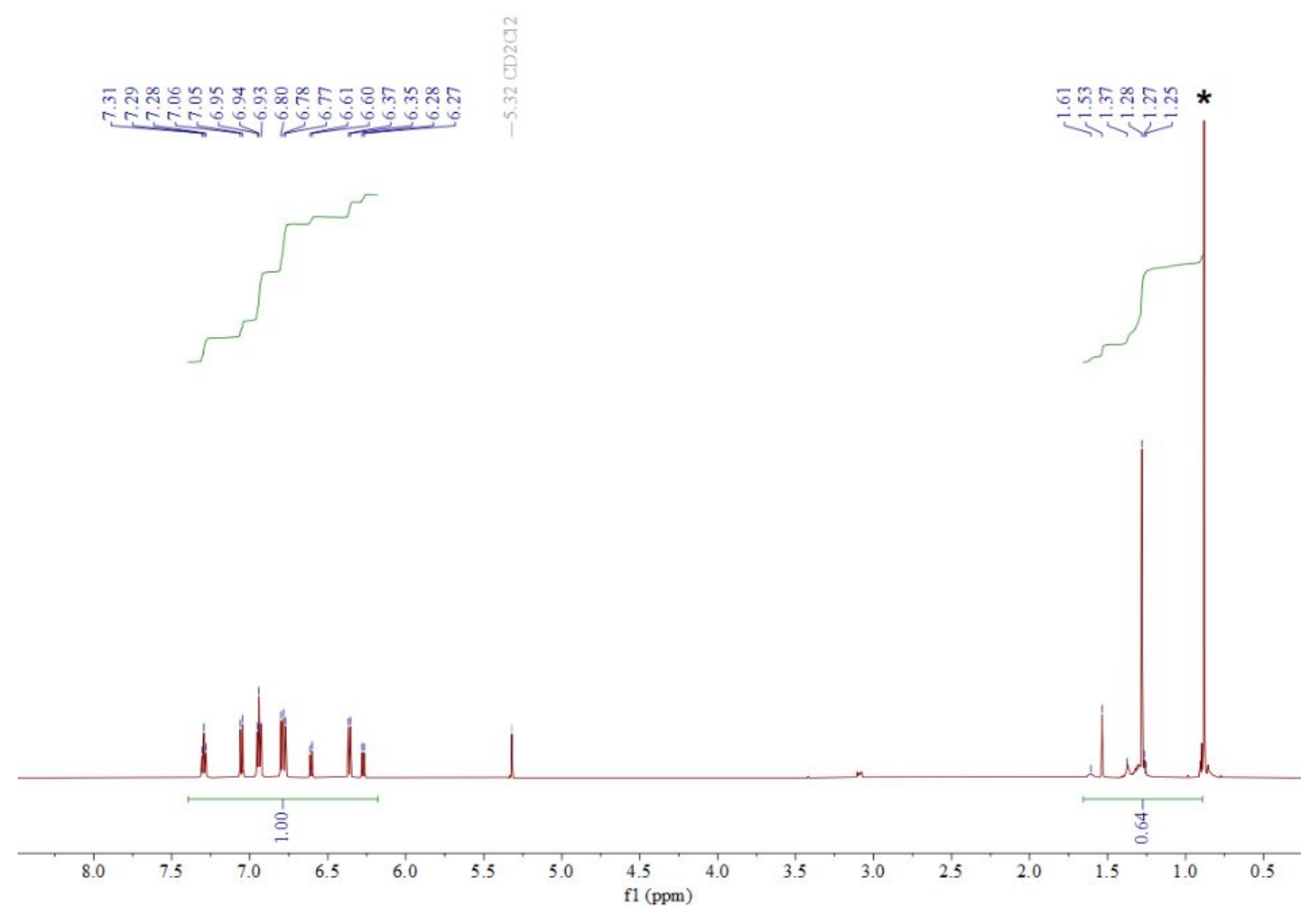

Figure S6. ${ }^{1} \mathrm{HNMR}$ of $\mathbf{C u}_{11}$ cluster dissolved in $\mathrm{CD}_{2} \mathrm{Cl}_{2}$. (* was residuary solvent peak of $\mathrm{n}$ hexane)

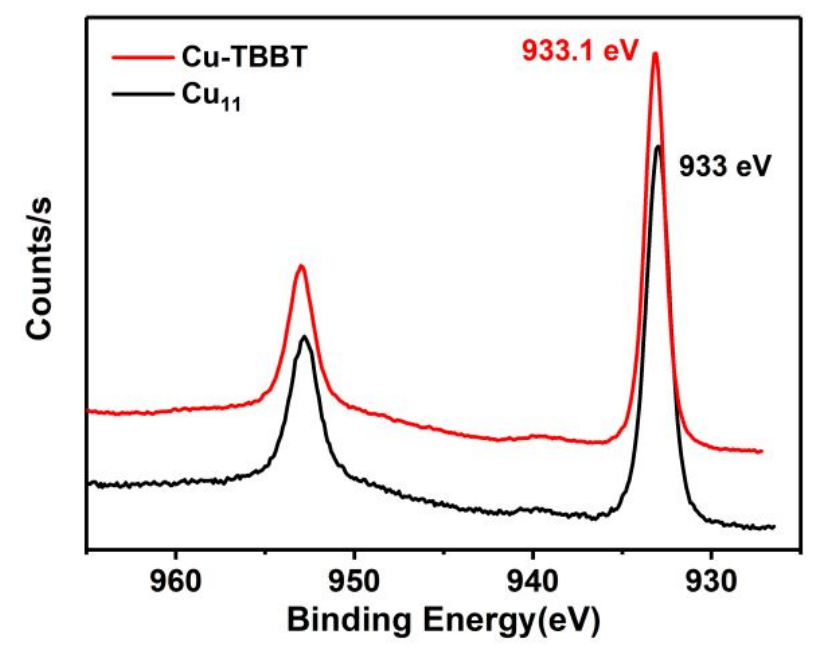

Figure S7. Compared with the binding energy for $\mathrm{Cu} 2 \mathrm{p}_{3 / 2}$ of $\mathrm{Cu}(\mathrm{I})-\mathrm{TBBT}$ complex and $\mathbf{C} \mathbf{u}_{11}$ cluster. 


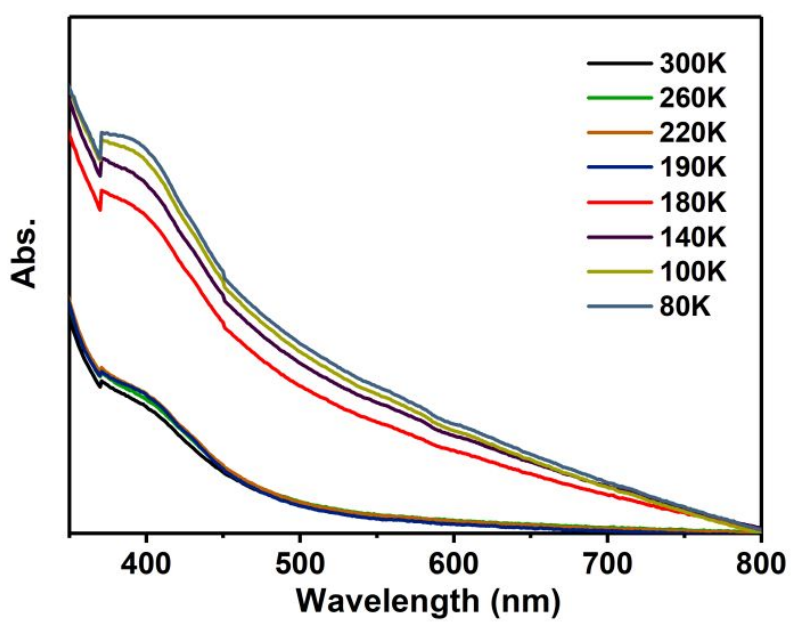

Figure S8. The temperature-depended UV-vis absorption spectra of $\mathbf{C} \mathbf{u}_{11}$ cluster in $\mathrm{CH}_{2} \mathrm{Cl}_{2}$ solution. Note: the sudden change in baseline at $180 \mathrm{~K}$ is induced by the solidification of $\mathrm{CHCl}_{2}$ solution.

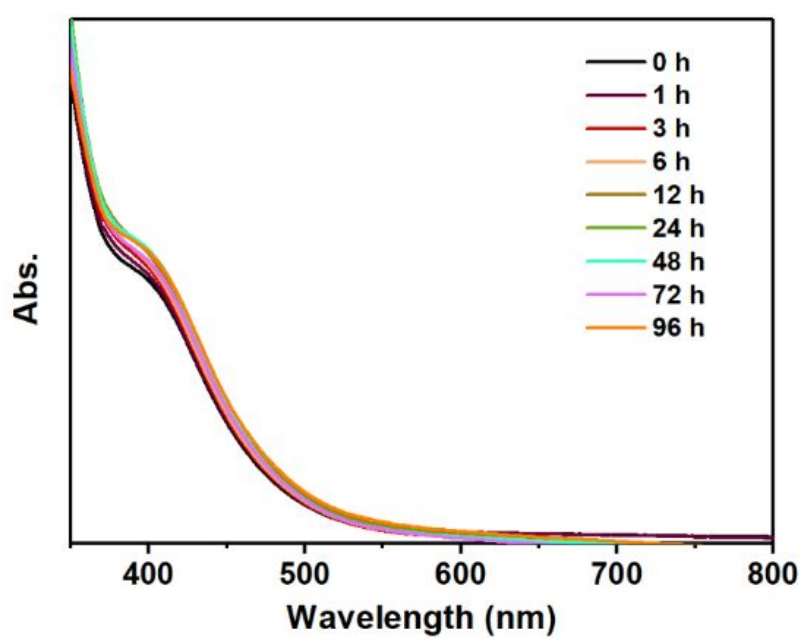

Figure S9. Time-dependent UV-vis absorption spectra of $\mathbf{C} \mathbf{u}_{11}$ cluster at room temperature in $\mathrm{CHCl}_{3}$.

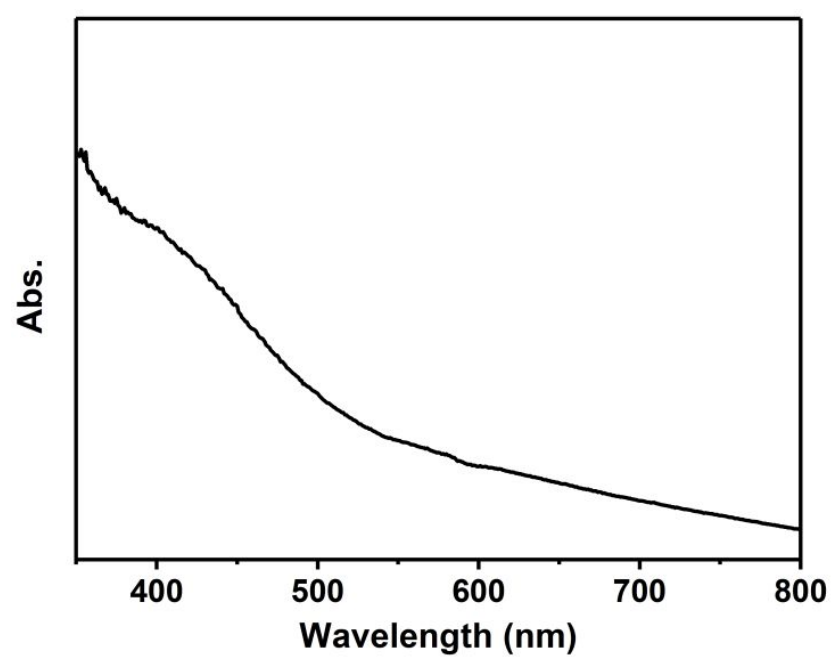

Figure S10. UV-vis optical absorption spectrum of $\mathbf{C} \mathbf{u}_{11}$ cluster in solid state. 


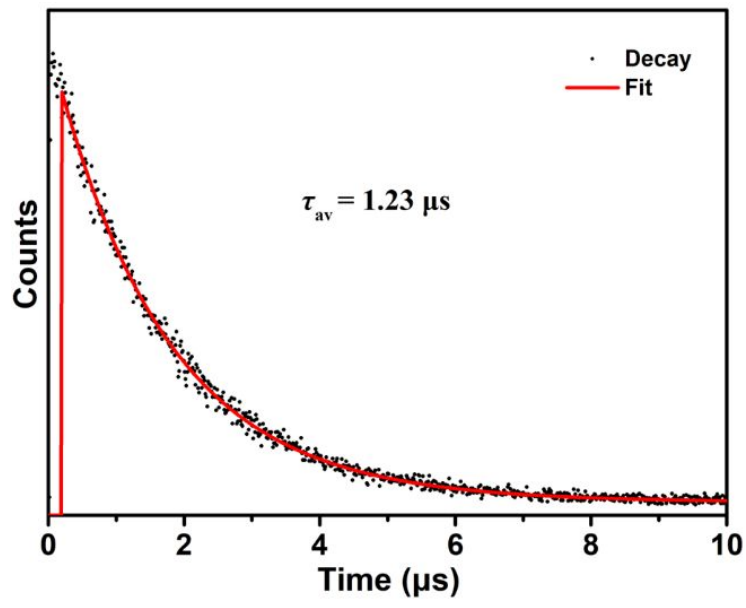

Figure S11. The emission lifetime of $\mathbf{C u}_{11}$ clusters in $\mathrm{CHCl}_{3}$ solution.

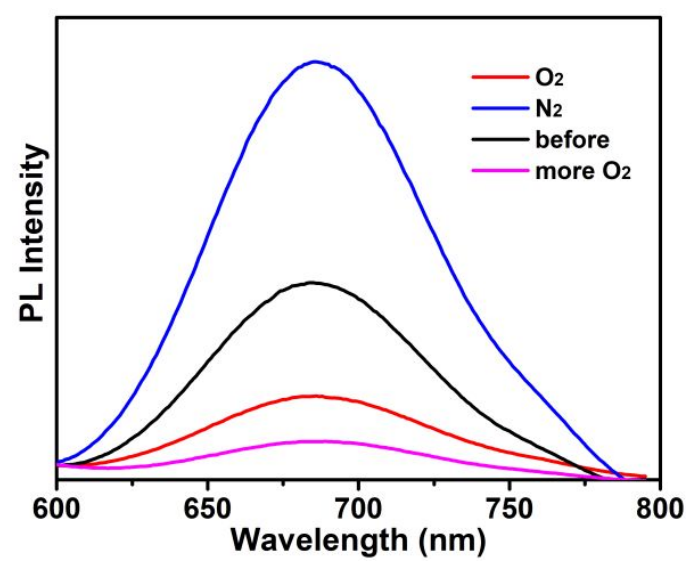

Figure S12. Emission spectra of the $\mathbf{C u}_{11}$ cluster in $\mathrm{CHCl}_{3}$ solution under $\mathrm{O}_{2}$ and $\mathrm{N}_{2}$ atmospheres (excited at $430 \mathrm{~nm}$ ).

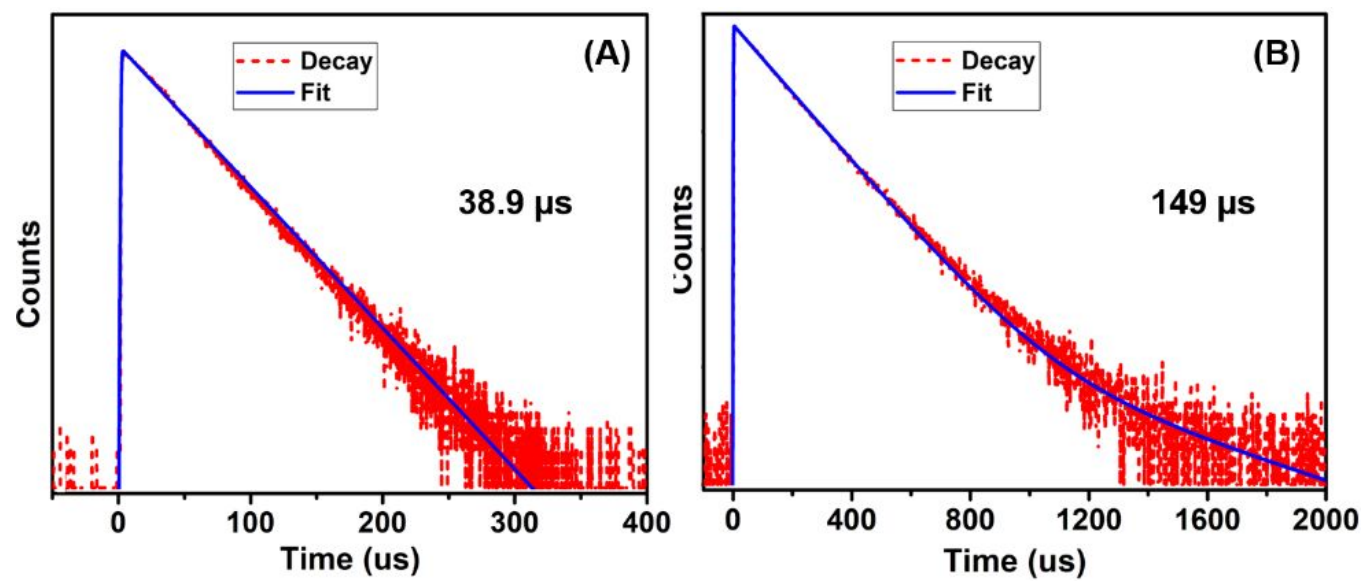

Figure S13. The emission decay lifetime of $\mathbf{C u}_{11}$ cluster at $180 \mathrm{~K}$ (A) and $80 \mathrm{~K}$ (B). 


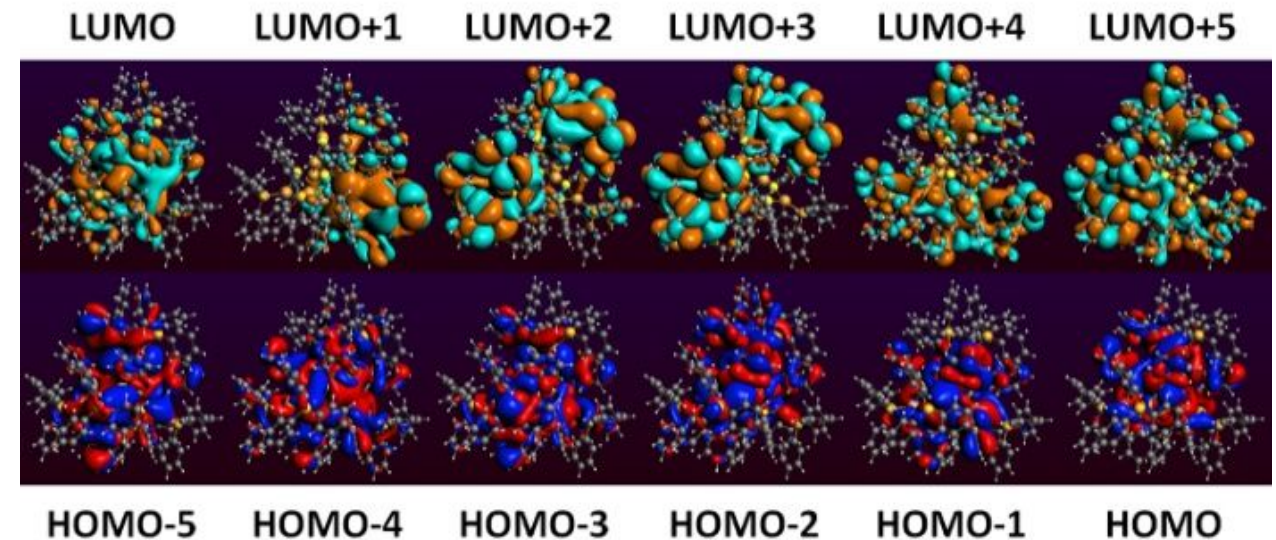

Figure S14. The electron densities of HOMOs and LUMOs in $\left[\mathrm{Cu}_{11}(\mathrm{SPh})_{9}\left(\mathrm{PPh}_{3}\right)_{6}\right]^{2+}$. 
Table S1. Crystal data and structure refinement for $\mathbf{C} \mathbf{u}_{11}$.

Identification code

Empirical formula

Formula weight

Temperature

Wavelength

Crystal system

Space group

Unit cell dimensions

Volume

Z

Density (calculated)

Absorption coefficient

$\mathrm{F}(000)$

Crystal size

Theta range for data collection

Index ranges

Reflections collected

Independent reflections

Completeness to theta $=55.290^{\circ}$

Absorption correction

Max. and min. transmission

Refinement method

Data / restraints / parameters

Goodness-of-fit on $\mathrm{F}^{2}$

Final $\mathrm{R}$ indices [I $>2 \operatorname{sigma}(\mathrm{I})]$

$\mathrm{R}$ indices (all data)

Largest diff. peak and hole

\section{$\mathrm{Cu}_{11}$}

$\mathrm{C}_{197} \mathrm{H}_{208} \mathrm{Cu}_{11} \mathrm{P}_{6} \mathrm{~S}_{9} \mathrm{~F}_{12} \mathrm{Sb}_{2}$

4220.58

$170.01 \mathrm{~K}$

$1.3418 \AA$

Monoclinic

P $2{ }_{1} / \mathrm{n}$

$\mathrm{a}=26.564(2) \AA$

$\alpha=90^{\circ}$

$\mathrm{b}=26.682(2) \AA$

$\mathrm{c}=33.045(3) \AA$

22448(3) $\AA^{3}$

4

$1.249 \mathrm{Mg} / \mathrm{cm}^{3}$

$0.323 \mathrm{~mm}^{-1}$

8612

$0.1 \times 0.03 \times 0.02 \mathrm{~mm}^{3}$

2.856 to $55.290^{\circ}$

$-32<=\mathrm{h}<=28,-32<=\mathrm{k}<=32$,

$-40<=1<=40$

233199

$42387[\mathrm{R}$ (int) $=0.2430]$

$98.1 \%$

Semi-empirical from equivalents

0.7508 and 0.2514

Full-matrix-block least-squares on

$$
\mathrm{F}^{2}
$$

42387 / 1182 / 2124

$$
1.326
$$

$\mathrm{R}_{1}=0.1948, \mathrm{wR} 2=0.4618$

$\mathrm{R}_{1}=0.2785, \mathrm{wR} 2=0.4984$

2.454 and -2.715 e. $\AA^{-3}$

Table S2 Photophysical data of $\mathbf{C} \mathbf{u}_{11}$ cluster at room temperature.

\begin{tabular}{cccccc}
\hline & $\lambda_{\text {ex }}(\mathrm{nm})$ & $\lambda_{\text {em }}(\mathrm{nm})$ & PLQY & $\tau_{\text {av }}(\mu \mathrm{s})$ & UV-vis Abs. $(\mathrm{nm})$ \\
\hline Solid & 450 & 675 & $22 \%$ & 1.3 & 400 \\
Solution & 430 & 685 & $7 \%$ & 1.23 & 400 \\
\hline
\end{tabular}


Table S3. Cartesian coordinates of the geometrically optimized $\mathrm{Cu}_{11}(\mathrm{SPh})_{9}\left(\mathrm{PPh}_{3}\right)_{6}$.

\begin{tabular}{|c|c|c|c|}
\hline Atom & $\mathbf{x}$ & $\mathbf{y}$ & $\mathbf{z}$ \\
\hline $\mathrm{Cu}$ & 7.15162 & 6.88763 & 30.51370 \\
\hline $\mathrm{Cu}$ & 7.29358 & 7.96634 & 27.72904 \\
\hline $\mathrm{Cu}$ & 3.99233 & 5.59434 & 32.86177 \\
\hline $\mathrm{Cu}$ & 7.59115 & 10.91835 & 30.73132 \\
\hline $\mathrm{Cu}$ & 9.42304 & 4.67489 & 28.20983 \\
\hline $\mathrm{Cu}$ & 5.33609 & 4.84221 & 29.26003 \\
\hline $\mathrm{Cu}$ & 4.48406 & 8.63749 & 30.45928 \\
\hline $\mathrm{Cu}$ & 6.75758 & 4.60926 & 25.73022 \\
\hline $\mathrm{Cu}$ & 5.27105 & 11.35298 & 27.75897 \\
\hline $\mathrm{Cu}$ & 1.56865 & 5.57807 & 29.96054 \\
\hline $\mathrm{Cu}$ & 4.32310 & 7.39491 & 27.79570 \\
\hline $\mathrm{S}$ & 7.62498 & 4.71452 & 29.80788 \\
\hline S & 8.20925 & 8.82597 & 29.72928 \\
\hline S & 5.32204 & 10.81738 & 30.06010 \\
\hline $\mathrm{S}$ & 4.85559 & 5.23325 & 27.00904 \\
\hline S & 5.47058 & 7.31138 & 32.11569 \\
\hline $\mathrm{S}$ & 3.53516 & 4.54817 & 30.78336 \\
\hline S & 8.32627 & 6.11064 & 26.67730 \\
\hline S & 5.51503 & 9.17244 & 26.84255 \\
\hline $\mathrm{S}$ & 2.63441 & 7.64966 & 29.38626 \\
\hline $\mathrm{P}$ & 11.31724 & 3.39090 & 28.37819 \\
\hline $\mathrm{P}$ & -0.70160 & 5.22091 & 29.87607 \\
\hline $\mathrm{P}$ & 9.03795 & 12.42910 & 31.68895 \\
\hline $\mathrm{P}$ & 5.12697 & 13.24041 & 26.47704 \\
\hline $\mathrm{P}$ & 6.81232 & 2.94087 & 24.16626 \\
\hline $\mathrm{P}$ & 3.31988 & 5.29861 & 35.03070 \\
\hline $\mathrm{C}$ & 4.70067 & 4.75491 & 36.12718 \\
\hline $\mathrm{C}$ & 6.51742 & 9.42319 & 24.25006 \\
\hline $\mathrm{H}$ & 7.44184 & 9.75514 & 24.72163 \\
\hline $\mathrm{C}$ & 5.22605 & 9.15160 & 34.19121 \\
\hline $\mathrm{H}$ & 4.16295 & 9.15727 & 33.95303 \\
\hline $\mathrm{C}$ & 4.22655 & 11.97031 & 30.92854 \\
\hline $\mathrm{C}$ & 2.02600 & 2.31587 & 26.51192 \\
\hline $\mathrm{H}$ & 1.89641 & 1.27338 & 26.80558 \\
\hline $\mathrm{C}$ & 4.14826 & 8.56766 & 23.03590 \\
\hline $\mathrm{H}$ & 3.22123 & 8.23285 & 22.56753 \\
\hline $\mathrm{C}$ & 9.99008 & 8.67571 & 29.49378 \\
\hline $\mathrm{C}$ & 8.46020 & 2.23927 & 23.72654 \\
\hline $\mathrm{C}$ & 5.83688 & 1.44115 & 24.62041 \\
\hline $\mathrm{C}$ & 7.80075 & 3.13251 & 33.59175 \\
\hline $\mathrm{H}$ & 7.65570 & 3.48463 & 34.61416 \\
\hline $\mathrm{C}$ & 12.55318 & 3.52989 & 27.01688 \\
\hline
\end{tabular}




\begin{tabular}{|c|c|c|c|}
\hline $\mathrm{C}$ & 10.69083 & 7.52666 & 29.89054 \\
\hline $\mathrm{H}$ & 10.14984 & 6.68876 & 30.33445 \\
\hline $\mathrm{C}$ & 13.36352 & 2.44905 & 26.63027 \\
\hline $\mathrm{H}$ & 13.23963 & 1.47484 & 27.10440 \\
\hline $\mathrm{C}$ & 5.23316 & 8.95634 & 22.24459 \\
\hline $\mathrm{C}$ & 3.34993 & 4.34658 & 26.56076 \\
\hline $\mathrm{C}$ & 1.07486 & 2.94269 & 25.70024 \\
\hline $\mathrm{C}$ & 11.61791 & 3.84343 & 31.12756 \\
\hline $\mathrm{H}$ & 10.52936 & 3.76490 & 31.15497 \\
\hline $\mathrm{C}$ & 9.89266 & 13.36547 & 30.34766 \\
\hline $\mathrm{C}$ & 10.39484 & 11.76466 & 32.74859 \\
\hline $\mathrm{C}$ & 10.36850 & -1.16233 & 28.40112 \\
\hline $\mathrm{H}$ & 10.14735 & -2.23054 & 28.38346 \\
\hline $\mathrm{C}$ & 12.07985 & 7.46562 & 29.73462 \\
\hline $\mathrm{H}$ & 12.61385 & 6.57042 & 30.05552 \\
\hline $\mathrm{C}$ & 3.15601 & 3.01216 & 26.94742 \\
\hline $\mathrm{H}$ & 3.89591 & 2.51602 & 27.57498 \\
\hline $\mathrm{C}$ & -0.32895 & 11.03957 & 28.37962 \\
\hline $\mathrm{C}$ & 7.81610 & 3.59000 & 31.20837 \\
\hline $\mathrm{C}$ & 3.36767 & 2.76524 & 31.05412 \\
\hline $\mathrm{C}$ & 13.71814 & 4.16661 & 32.29156 \\
\hline $\mathrm{H}$ & 14.26863 & 4.34032 & 33.21741 \\
\hline $\mathrm{C}$ & 7.67895 & 12.80791 & 25.42501 \\
\hline $\mathrm{H}$ & 7.88709 & 12.37190 & 26.40481 \\
\hline $\mathrm{C}$ & 12.30398 & 3.70766 & 29.90747 \\
\hline $\mathrm{C}$ & 6.10968 & 8.37526 & 33.42505 \\
\hline $\mathrm{C}$ & -0.96017 & 6.71998 & 27.51212 \\
\hline $\mathrm{H}$ & 0.04958 & 7.06746 & 27.73396 \\
\hline $\mathrm{C}$ & 7.95241 & 9.14103 & 34.80919 \\
\hline $\mathrm{H}$ & 9.01900 & 9.13935 & 35.03838 \\
\hline $\mathrm{C}$ & 5.70406 & 9.89819 & 35.26993 \\
\hline $\mathrm{H}$ & 5.00210 & 10.48296 & 35.86606 \\
\hline $\mathrm{C}$ & 11.26307 & 13.66595 & 30.38604 \\
\hline $\mathrm{H}$ & 11.87466 & 13.32326 & 31.22067 \\
\hline $\mathrm{C}$ & 7.64361 & 4.02873 & 32.53001 \\
\hline $\mathrm{H}$ & 7.37478 & 5.06819 & 32.72404 \\
\hline $\mathrm{C}$ & 1.25620 & 4.27904 & 25.33142 \\
\hline $\mathrm{H}$ & 0.51503 & 4.77976 & 24.70707 \\
\hline $\mathrm{C}$ & 12.71298 & 4.77190 & 26.38281 \\
\hline $\mathrm{H}$ & 12.07426 & 5.61228 & 26.66055 \\
\hline $\mathrm{C}$ & -1.62922 & 7.19752 & 26.38135 \\
\hline $\mathrm{H}$ & -1.13862 & 7.92223 & 25.73033 \\
\hline $\mathrm{C}$ & 12.08135 & 9.68754 & 28.78614 \\
\hline $\mathrm{H}$ & 12.61701 & 10.53841 & 28.36257 \\
\hline
\end{tabular}




\begin{tabular}{|c|c|c|c|}
\hline $\mathrm{C}$ & 4.24095 & 8.61043 & 24.42953 \\
\hline $\mathrm{H}$ & 3.38986 & 8.31699 & 25.04577 \\
\hline $\mathrm{C}$ & 10.69423 & 9.75490 & 28.93369 \\
\hline $\mathrm{H}$ & 10.15440 & 10.65181 & 28.62830 \\
\hline $\mathrm{C}$ & 9.12242 & 13.80755 & 29.25698 \\
\hline $\mathrm{H}$ & 8.05453 & 13.57778 & 29.21363 \\
\hline $\mathrm{C}$ & 12.78033 & 8.54371 & 29.18575 \\
\hline $\mathrm{C}$ & 6.39268 & 13.29289 & 25.13618 \\
\hline $\mathrm{C}$ & 2.38832 & 4.97923 & 25.75631 \\
\hline $\mathrm{H}$ & 2.53856 & 6.01679 & 25.45632 \\
\hline $\mathrm{C}$ & 7.06619 & 9.89030 & 35.58907 \\
\hline $\mathrm{C}$ & 7.47982 & 8.38482 & 33.73214 \\
\hline $\mathrm{H}$ & 8.17357 & 7.79336 & 33.13272 \\
\hline $\mathrm{C}$ & 4.40738 & 2.05020 & 31.66828 \\
\hline $\mathrm{H}$ & 5.31652 & 2.56535 & 31.97911 \\
\hline $\mathrm{C}$ & 2.07612 & 13.06991 & 31.12640 \\
\hline $\mathrm{H}$ & 1.06119 & 13.21936 & 30.75616 \\
\hline $\mathrm{C}$ & 3.11149 & 0.00462 & 31.49207 \\
\hline $\mathrm{C}$ & 6.73377 & 3.30613 & 21.33199 \\
\hline $\mathrm{H}$ & 7.69870 & 2.80054 & 21.29120 \\
\hline $\mathrm{C}$ & 11.27858 & 8.24402 & 24.81722 \\
\hline $\mathrm{H}$ & 12.12617 & 8.85804 & 25.12617 \\
\hline $\mathrm{C}$ & 9.08943 & 6.68788 & 24.04967 \\
\hline $\mathrm{H}$ & 8.21917 & 6.10257 & 23.74837 \\
\hline $\mathrm{C}$ & 9.38250 & 6.85362 & 25.41051 \\
\hline $\mathrm{C}$ & 1.94731 & 12.37514 & 24.01878 \\
\hline $\mathrm{H}$ & 1.68449 & 11.56620 & 23.33549 \\
\hline $\mathrm{C}$ & 8.31373 & 1.36197 & 32.02991 \\
\hline $\mathrm{H}$ & 8.58066 & 0.32395 & 31.82603 \\
\hline $\mathrm{C}$ & 12.50827 & 10.72732 & 34.27891 \\
\hline $\mathrm{H}$ & 13.33096 & 10.32624 & 34.87273 \\
\hline $\mathrm{C}$ & 9.60698 & 3.02428 & 23.92500 \\
\hline $\mathrm{H}$ & 9.51662 & 4.01617 & 24.37135 \\
\hline $\mathrm{C}$ & 2.91588 & 12.16772 & 30.46820 \\
\hline $\mathrm{H}$ & 2.55335 & 11.62064 & 29.59759 \\
\hline $\mathrm{C}$ & 5.42699 & 9.04122 & 25.04519 \\
\hline $\mathrm{C}$ & 2.53055 & 13.77672 & 32.24427 \\
\hline $\mathrm{C}$ & 8.59371 & 0.95476 & 23.16957 \\
\hline $\mathrm{H}$ & 7.71370 & 0.32675 & 23.02426 \\
\hline $\mathrm{C}$ & 12.08583 & 12.04664 & 34.47175 \\
\hline $\mathrm{H}$ & 12.57619 & 12.67627 & 35.21544 \\
\hline $\mathrm{C}$ & 0.72419 & 10.75918 & 27.50382 \\
\hline $\mathrm{H}$ & 0.84428 & 11.33702 & 26.58594 \\
\hline $\mathrm{C}$ & 1.49747 & 8.99060 & 28.97477 \\
\hline
\end{tabular}




\begin{tabular}{|c|c|c|c|}
\hline $\mathrm{C}$ & -0.91583 & 5.98544 & 32.55684 \\
\hline $\mathrm{H}$ & -0.05167 & 5.33179 & 32.70012 \\
\hline $\mathrm{C}$ & -1.47136 & 6.64462 & 33.65551 \\
\hline $\mathrm{H}$ & -1.03972 & 6.49968 & 34.64635 \\
\hline $\mathrm{C}$ & -2.57399 & 7.48788 & 33.48326 \\
\hline $\mathrm{H}$ & -3.00615 & 8.00544 & 34.34090 \\
\hline $\mathrm{C}$ & -3.12120 & 7.66427 & 32.20990 \\
\hline $\mathrm{H}$ & -3.98079 & 8.32160 & 32.06990 \\
\hline $\mathrm{C}$ & -2.57879 & 6.99333 & 31.10926 \\
\hline $\mathrm{H}$ & -3.01673 & 7.13407 & 30.12112 \\
\hline $\mathrm{C}$ & -1.47459 & 6.14366 & 31.27518 \\
\hline $\mathrm{C}$ & 11.03515 & 12.56512 & 33.71156 \\
\hline $\mathrm{H}$ & 10.71002 & 13.59338 & 33.87247 \\
\hline $\mathrm{C}$ & 1.94119 & 7.73133 & 35.02905 \\
\hline $\mathrm{H}$ & 1.75547 & 7.50004 & 33.97752 \\
\hline $\mathrm{C}$ & -2.91987 & 6.75027 & 26.08813 \\
\hline $\mathrm{H}$ & -3.44082 & 7.12082 & 25.20404 \\
\hline $\mathrm{C}$ & -1.04282 & 2.56871 & 29.05405 \\
\hline $\mathrm{H}$ & -0.43976 & 2.87272 & 28.19537 \\
\hline $\mathrm{C}$ & 10.07596 & 1.05536 & 27.47233 \\
\hline $\mathrm{H}$ & 9.63026 & 1.71128 & 26.72069 \\
\hline $\mathrm{C}$ & 6.01747 & 0.90869 & 25.90864 \\
\hline $\mathrm{H}$ & 6.68120 & 1.40945 & 26.61723 \\
\hline $\mathrm{C}$ & 10.47867 & 7.64297 & 25.79250 \\
\hline $\mathrm{H}$ & 10.69782 & 7.79595 & 26.85009 \\
\hline $\mathrm{C}$ & 2.70522 & 6.85156 & 35.81410 \\
\hline $\mathrm{C}$ & 12.32335 & 4.06316 & 32.31247 \\
\hline $\mathrm{H}$ & 11.77990 & 4.15240 & 33.25399 \\
\hline $\mathrm{C}$ & 9.70952 & 14.55919 & 28.23652 \\
\hline $\mathrm{H}$ & 9.09856 & 14.90946 & 27.40391 \\
\hline $\mathrm{C}$ & 11.07465 & 14.85973 & 28.28423 \\
\hline $\mathrm{H}$ & 11.53369 & 15.44674 & 27.48750 \\
\hline $\mathrm{C}$ & 14.40487 & 4.04591 & 31.08063 \\
\hline $\mathrm{H}$ & 15.49269 & 4.12580 & 31.05740 \\
\hline $\mathrm{C}$ & 8.42748 & 13.41559 & 23.20411 \\
\hline $\mathrm{H}$ & 9.21756 & 13.46589 & 22.45325 \\
\hline $\mathrm{C}$ & 0.85535 & 2.01938 & 34.71501 \\
\hline $\mathrm{H}$ & 0.77307 & 1.16481 & 34.04271 \\
\hline $\mathrm{C}$ & 2.07975 & 0.71950 & 30.87699 \\
\hline $\mathrm{H}$ & 1.16482 & 0.21209 & 30.56714 \\
\hline $\mathrm{C}$ & 13.70434 & 3.81395 & 29.89277 \\
\hline $\mathrm{H}$ & 14.25129 & 3.71766 & 28.95491 \\
\hline $\mathrm{C}$ & -1.58442 & 5.79725 & 28.36432 \\
\hline $\mathrm{C}$ & 6.41519 & 9.38256 & 22.85758 \\
\hline
\end{tabular}


H

C

$\mathrm{H}$

C

$\mathrm{H}$

C

$\mathrm{H}$

C

$\mathrm{H}$

C

$\mathrm{H}$

C

$\mathrm{H}$

C

$\mathrm{H}$

C

$\mathrm{H}$

C

$\mathrm{H}$

C

$\mathrm{H}$

C

$\mathrm{H}$

C

C

$\mathrm{H}$

C

$\mathrm{H}$

C

$\mathrm{H}$

C

$\mathrm{H}$

C

$\mathrm{H}$

C

$\mathrm{H}$

C

$\mathrm{H}$

C

$\mathrm{H}$

C

$\mathrm{H}$

C

$\mathrm{H}$
7.26847

8.14916

8.28677

4.27373

5.08658

6.13188

5.13429

10.81698

10.32234

2.20509

1.39499

14.49342

15.24961

14.32912

14.95606

$-0.46363$

$-1.28123$

2.64496

2.91293

10.98942

11.97174

9.79220

9.12284

10.95001

3.83460

4.20508

6.87286

7.71382

11.52380

12.20098

9.85348

9.94809

11.87279

12.19745

3.15604

3.82369

8.69257

9.68849

10.86545

11.74890

1.66963

1.26822

$-2.12598$

$-2.37757$
9.68720

22.25037

2.24839

1.90179

30.96255

0.67612

0.13183

13.82815

14.19430

10.43879

9.80672

2.09408

2.64698

3.86115

3.98996

2.61726

1.77349

10.29503

10.50424

14.45506

15.27346

1.27026

0.89250

$-0.31136$

$-0.71284$

1.58365

13.57204

14.11811

4.00066

3.71040

0.72563

1.12257

0.47582

$-0.52080$

9.92549

8.89600

12.32401

11.47140

12.87529

12.49818

2.54248

3.16148

9.21470

10.13283

3.08881

3.79637
29.93797

31.88511

32.36772

23.86424

23.61948

32.56841

31.83046

30.65571

30.18022

25.01655

24.24089

25.63437

25.34222

29.55578

30.24721

25.75365

26.42211

22.99071

22.70380

27.45159

26.68978

28.43909

32.70395

33.57280

37.73843

38.36984

29.38951

30.14595

22.80333

22.36944

33.32688

33.17064

24.71448

24.57255

24.46662

24.70290

23.55441

23.71520

36.92319

37.35505

31.15893

31.94872 


\begin{tabular}{|c|c|c|c|}
\hline $\mathrm{C}$ & 5.86201 & 5.54830 & 36.17107 \\
\hline $\mathrm{H}$ & 5.92291 & 6.46543 & 35.58128 \\
\hline $\mathrm{C}$ & 4.85112 & 4.16300 & 22.59966 \\
\hline $\mathrm{H}$ & 4.34604 & 4.32984 & 23.55390 \\
\hline $\mathrm{C}$ & 9.90135 & 7.28422 & 23.08026 \\
\hline $\mathrm{H}$ & 9.66719 & 7.14103 & 22.02433 \\
\hline $\mathrm{C}$ & 11.84906 & 14.40783 & 29.35614 \\
\hline $\mathrm{H}$ & 12.91472 & 14.63870 & 29.39746 \\
\hline $\mathrm{C}$ & 1.07962 & 13.45976 & 24.19215 \\
\hline $\mathrm{H}$ & 0.13623 & 13.49905 & 23.64582 \\
\hline $\mathrm{C}$ & 4.48961 & -0.89759 & 25.39397 \\
\hline $\mathrm{H}$ & 3.96959 & -1.80919 & 25.69175 \\
\hline $\mathrm{C}$ & 2.43463 & 8.34763 & 37.70856 \\
\hline $\mathrm{H}$ & 2.63011 & 8.58558 & 38.75530 \\
\hline $\mathrm{C}$ & 4.25458 & 4.59761 & 21.41543 \\
\hline $\mathrm{H}$ & 3.28534 & 5.09677 & 21.45048 \\
\hline $\mathrm{C}$ & 0.08634 & 3.26181 & 36.64445 \\
\hline $\mathrm{H}$ & -0.59977 & 3.37530 & 37.48517 \\
\hline $\mathrm{C}$ & 6.09197 & 3.50098 & 22.56492 \\
\hline $\mathrm{C}$ & 4.68138 & 12.67077 & 32.05214 \\
\hline $\mathrm{H}$ & 5.69890 & 12.51984 & 32.41177 \\
\hline $\mathrm{C}$ & 0.44423 & 9.27757 & 29.85707 \\
\hline $\mathrm{H}$ & 0.33369 & 8.69854 & 30.77450 \\
\hline $\mathrm{C}$ & 10.99800 & 8.06335 & 23.45849 \\
\hline $\mathrm{C}$ & 3.51980 & 13.37237 & 25.57971 \\
\hline $\mathrm{C}$ & 2.95107 & 7.17049 & 37.15906 \\
\hline $\mathrm{H}$ & 3.55147 & 6.50247 & 37.77776 \\
\hline $\mathrm{C}$ & 6.13698 & 3.75654 & 20.15022 \\
\hline $\mathrm{H}$ & 6.64278 & 3.59864 & 19.19655 \\
\hline $\mathrm{C}$ & -2.88178 & 5.34578 & 28.06090 \\
\hline $\mathrm{H}$ & -3.37247 & 4.61672 & 28.70668 \\
\hline $\mathrm{C}$ & 1.63390 & 9.73746 & 27.79518 \\
\hline $\mathrm{H}$ & 2.45950 & 9.52573 & 27.11389 \\
\hline $\mathrm{C}$ & 7.15964 & 14.31780 & 34.82295 \\
\hline $\mathrm{H}$ & 6.68675 & 14.00302 & 35.75442 \\
\hline $\mathrm{C}$ & 7.23625 & 15.67745 & 34.49969 \\
\hline $\mathrm{H}$ & 6.82204 & 16.42575 & 35.17687 \\
\hline $\mathrm{C}$ & 7.84974 & 16.07204 & 33.30910 \\
\hline $\mathrm{H}$ & 7.91958 & 17.13043 & 33.05357 \\
\hline $\mathrm{C}$ & 8.39349 & 15.11618 & 32.44395 \\
\hline $\mathrm{H}$ & 8.88238 & 15.43868 & 31.52488 \\
\hline $\mathrm{C}$ & 8.32146 & 13.75273 & 32.76414 \\
\hline $\mathrm{C}$ & 7.69174 & 13.35966 & 33.95901 \\
\hline $\mathrm{H}$ & 7.62822 & 12.30143 & 34.22334 \\
\hline
\end{tabular}




\begin{tabular}{|c|c|c|c|}
\hline $\mathrm{C}$ & 5.35334 & -0.25809 & 26.29037 \\
\hline $\mathrm{H}$ & 5.50492 & -0.66565 & 27.29089 \\
\hline $\mathrm{C}$ & -2.30128 & 0.85891 & 30.22313 \\
\hline $\mathrm{H}$ & -2.67912 & -0.16286 & 30.28072 \\
\hline $\mathrm{C}$ & 1.96624 & 4.08611 & 35.34544 \\
\hline $\mathrm{C}$ & -3.54411 & 5.82159 & 26.92795 \\
\hline $\mathrm{H}$ & -4.55024 & 5.46632 & 26.70107 \\
\hline $\mathrm{C}$ & 1.42250 & 8.90437 & 35.58193 \\
\hline $\mathrm{H}$ & 0.83160 & 9.58093 & 34.96279 \\
\hline $\mathrm{C}$ & 11.23093 & -0.64178 & 29.36900 \\
\hline $\mathrm{H}$ & 11.68678 & -1.30159 & 30.10872 \\
\hline $\mathrm{C}$ & 4.29246 & -0.36367 & 24.11814 \\
\hline $\mathrm{H}$ & 3.61766 & -0.85609 & 23.41650 \\
\hline $\mathrm{C}$ & 4.64332 & 3.57644 & 36.88608 \\
\hline $\mathrm{H}$ & 3.75050 & 2.95168 & 36.86007 \\
\hline $\mathrm{C}$ & 4.98970 & 14.95963 & 28.68739 \\
\hline $\mathrm{H}$ & 4.65649 & 14.07348 & 29.23076 \\
\hline $\mathrm{C}$ & 5.08102 & 16.18682 & 29.35109 \\
\hline $\mathrm{H}$ & 4.81790 & 16.24736 & 30.40758 \\
\hline $\mathrm{C}$ & 5.51171 & 17.32509 & 28.66503 \\
\hline $\mathrm{H}$ & 5.58411 & 18.28216 & 29.18383 \\
\hline $\mathrm{C}$ & 5.85342 & 17.23651 & 27.31142 \\
\hline $\mathrm{H}$ & 6.19302 & 18.12237 & 26.77289 \\
\hline $\mathrm{C}$ & 5.75940 & 16.01409 & 26.64289 \\
\hline $\mathrm{H}$ & 6.03017 & 15.95457 & 25.58809 \\
\hline $\mathrm{C}$ & 5.32041 & 14.86625 & 27.32593 \\
\hline $\mathrm{C}$ & 1.07666 & 4.22229 & 36.42530 \\
\hline $\mathrm{H}$ & 1.15426 & 5.07920 & 37.09545 \\
\hline $\mathrm{C}$ & 8.13901 & 1.79803 & 33.34721 \\
\hline $\mathrm{C}$ & 7.14623 & 13.88672 & 22.90414 \\
\hline $\mathrm{H}$ & 6.93344 & 14.30455 & 21.91908 \\
\hline $\mathrm{C}$ & 13.68195 & 4.93697 & 25.39006 \\
\hline $\mathrm{H}$ & 13.79587 & 5.90638 & 24.90330 \\
\hline $\mathrm{C}$ & -0.02361 & 2.15836 & 35.79235 \\
\hline $\mathrm{H}$ & -0.79689 & 1.40927 & 35.96809 \\
\hline $\mathrm{C}$ & -1.34688 & 3.49700 & 30.06667 \\
\hline $\mathrm{C}$ & -2.59715 & 1.77365 & 31.23559 \\
\hline $\mathrm{H}$ & -3.20874 & 1.47007 & 32.08647 \\
\hline $\mathrm{C}$ & 1.84318 & 2.98146 & 34.48716 \\
\hline $\mathrm{H}$ & 2.52001 & 2.87072 & 33.63797 \\
\hline $\mathrm{C}$ & 4.89608 & 4.39668 & 20.18852 \\
\hline $\mathrm{H}$ & 4.42945 & 4.73952 & 19.26393 \\
\hline $\mathrm{C}$ & 1.42848 & 14.49409 & 25.06355 \\
\hline $\mathrm{H}$ & 0.75799 & 15.34355 & 25.20177 \\
\hline
\end{tabular}




$\begin{array}{lccc}\mathrm{C} & 6.93633 & 5.17585 & 36.98049 \\ \mathrm{H} & 7.82523 & 5.80688 & 37.02019 \\ \mathrm{C} & -1.52365 & 1.26069 & 29.13089 \\ \mathrm{H} & -1.29659 & 0.55279 & 28.33220 \\ \mathrm{C} & 5.72882 & 3.20131 & 37.68502 \\ \mathrm{H} & 5.67277 & 2.28506 & 38.27433 \\ \mathrm{C} & 4.96424 & 0.79971 & 23.72879 \\ \mathrm{H} & 4.80700 & 1.20668 & 22.72957 \\ \mathrm{H} & 7.43504 & 10.46248 & 36.44141 \\ \mathrm{H} & 13.86426 & 8.49535 & 29.07646 \\ \mathrm{H} & 8.26774 & 1.10313 & 34.17785 \\ \mathrm{H} & 3.01077 & -1.06747 & 31.66608 \\ \mathrm{H} & 0.19921 & 2.39302 & 25.35279 \\ \mathrm{H} & -1.04093 & 11.83205 & 28.14624 \\ \mathrm{H} & 1.87274 & 14.48317 & 32.75216 \\ \mathrm{H} & 11.62720 & 8.53076 & 22.69999 \\ \mathrm{H} & 5.15779 & 8.92705 & 21.15706\end{array}$

